\title{
Non-polynomial Third Order Equations which Pass the Painlevé Test
}

Article · June 2005

DOI: 10.1515/zna-2005-0601

\section{CITATIONS}

10

2 authors:

\section{Fahd Jarad}

Cankaya University

42 PUBLICATIONS 612 CITATIONS

SEE PROFILE

Some of the authors of this publication are also working on these related projects: 


\title{
Non-polynomial fourth order equations which pass the Painlevé test ${ }^{1}$
}

\author{
F. JRAD $D^{\dagger}$ and $U . M U \breve{G} A N^{\ddagger}$ \\ † Cankaya University, Department of Mathematics and Computer Sciences, \\ 06530 Balgat, Ankara, Turkey \\ E-mail: fahd@cankaya.edu.tr \\ $\ddagger$ Bilkent University, Department of Mathematics, \\ 06800 Bilkent, Ankara, Turkey \\ E-mail: mugan@fen.bilkent.edu.tr
}

December 2004, revised February 2005

\begin{abstract}
The singular point analysis of fourth order ordinary differential equations in the nonpolynomial class are presented. Some new fourth order ordinary differential equations which pass the Painlevé test as well as the known ones are found.
\end{abstract}

Key words: Painlevé equations, Painlevé test.

\section{Introduction}

Painlevé and his school [1-3] studied the certain class of second order ordinary differential equations (ODE) and found fifty canonical equations whose solutions have no movable critical points. This property is known as the Painlevé property. Distinguished among these fifty equations are six Painlevé equations, PI-PVI. The six Painlevé transcendents are regarded as nonlinear special functions.

The fourth order equations of Painlevé type

$$
y^{(4)}=F\left(z, y, y^{\prime}, y^{\prime \prime}, y^{\prime \prime \prime}\right),
$$

where $F$ is polynomial in $y$ and its derivatives, were considered in $[4,5,6,7,8,9,10,11,12]$. Third order polynomial type equations with Painlevé property were investigated in $[4,5,6$, 11, 13]. Non-polynomial third order equations of Painlevé type were studied in $[14,15,16]$. Some of the fourth order non-polynomial equations possing Painlevé property were introduced in $[10,17,18,19,20,21]$.

In this article, we consider the simplified equation associated with the following fourth order differential equation

$$
y^{(4)}=a_{1} \frac{y^{\prime} y^{\prime \prime \prime}}{y}+a_{2} \frac{\left(y^{\prime \prime}\right)^{2}}{y}+a_{3} \frac{\left(y^{\prime}\right)^{2} y^{\prime \prime}}{y^{2}}+a_{4} \frac{\left(y^{\prime}\right)^{4}}{y^{3}}+F\left(z, y, y^{\prime}, y^{\prime \prime}, y^{\prime \prime \prime}\right),
$$

where $a_{j}, j=1, \ldots, 4$ are constants are not all zero. $F$ may contain the leading terms, but all the terms of $F$ are of order $\epsilon^{-3}$ or greater if we let $z=\zeta_{0}+\epsilon t$ where $\zeta_{0}$ is a constant, $\epsilon$ is a small parameter and $t$ is the new independent variable, and the coefficients in $F$ are locally

${ }^{1}$ Zeitschrift für Naturforschung A, 60a, 387-400, (2005) 
analytic functions of $z$. The equation of type (1.2) can be obtained by differentiating twice the leading terms of the third (or fourth) Painlevé equation and adding the terms of order -5 or greater as $z \rightarrow z_{0}$ (i.e. in the neighborhood of the movable pole $z_{0}$ ) with analytic coefficients in $z$ such that: i. $y=0, \infty$ are the only singular values of equation in $y$, ii. The additional terms are of order $\epsilon^{-4}$ or greater, if one lets $z=\zeta_{0}+\epsilon t$

If we let, $z=\zeta_{0}+\epsilon t$ and take the limit as $\epsilon \rightarrow 0$, (1.2) yields the following "reduced" equation:

$$
\dddot{y}=a_{1} \frac{\ddot{y} \dddot{y}}{y}+a_{2} \frac{(\ddot{y})^{2}}{y}+a_{3} \frac{(\dot{y})^{2} \ddot{y}}{y^{2}}+a_{4} \frac{(\dot{y})^{4}}{y^{3}},
$$

where $\cdot=d / d t$. Substituting $y \cong y_{0}\left(t-t_{0}\right)^{\alpha}$ into equation (1.3) gives

$$
\left(a_{1}+a_{2}+a_{3}+a_{4}-1\right) \alpha^{3}-\left(3 a_{1}+2 a_{2}+a_{3}-6\right) \alpha^{2}+\left(2 a_{1}+a_{2}-11\right) \alpha+6=0 .
$$

Depending on the coefficients of (1.4), we have the following three cases. In the case of single branch, let

$$
a_{1}+a_{2}+a_{3}+a_{4}-1=0, \quad 3 a_{1}+2 a_{2}+a_{3}-6=0, \quad 2 a_{1}+a_{2}-11 \neq 0,
$$

and the root of (1.4) be $\alpha=n \in \mathbb{Z}-\{0\}$. Substituting

$$
y \cong y_{0}\left(t-t_{0}\right)^{\alpha}+\beta\left(t-t_{0}\right)^{r+\alpha},
$$

into (1.3), we obtain the following equation for the Fuchs indices:

$$
r(r+1)\left\{r^{2}-\left[\left(a_{1}-4\right) n+7\right] r+6\right\}=0 .
$$

So, the Fuchs indices are $r_{0}=-1, r_{1}=0, r_{2}$ and $r_{3}$ such that

$$
r_{2}+r_{3}=\left(a_{1}-4\right) n+7 \quad \text { and } \quad r_{2} r_{3}=6 .
$$

In order to have distinct indices, (1.8.b) implies that $\left(r_{2}, r_{3}\right)=(1,6),(2,3),(-2,-3)$. From the equations (1.4), (1.5), and (1.8), one gets the following 3 cases for $\left(a_{1}, a_{2}, a_{3}, a_{4}\right)$ :

$$
\begin{aligned}
& \text { 1. }\left(a_{1}, a_{2}, a_{3}, a_{4}\right)=\left(4,3-\frac{6}{n},-12+\frac{12}{n}, 6-\frac{6}{n}\right), \\
& \text { 2. }\left(a_{1}, a_{2}, a_{3}, a_{4}\right)=\left(4-\frac{2}{n}, 3-\frac{2}{n},-12+\frac{10}{n}, 6-\frac{6}{n}\right), \\
& \text { 3. }\left(a_{1}, a_{2}, a_{3}, a_{4}\right)=\left(4-\frac{12}{n}, 3+\frac{18}{n},-12,6-\frac{6}{n}\right),
\end{aligned}
$$

respectively.

In the case of double branch, let

$$
a_{1}+a_{2}+a_{3}+a_{4}-1=0, \quad 3 a_{1}+2 a_{2}+a_{3}-6 \neq 0,
$$

and the roots of (1.4) be $\alpha_{1}=n$ and $\alpha_{2}=m$ such that $n \neq m$, and $n, m \in \mathbb{Z}-\{0\}$. Then the equation (1.4) implies that

$$
3 a_{1}+2 a_{2}+a_{3}-6=-\frac{6}{n m}, \quad 11-2 a_{1}-a_{2}=6\left(\frac{1}{n}+\frac{1}{m}\right) .
$$


Similarly, substituting (1.6) into (1.3) gives the following equations for the Fuchs indices $r_{j i}, j=$ $1,2, i=0,1,2,3$

$$
\begin{aligned}
& r_{1}\left(r_{1}+1\right)\left\{r_{1}^{2}-\left[\left(a_{1}-4\right) n+7\right] r_{1}+6-\frac{6 n}{m}\right\}=0, \\
& r_{2}\left(r_{2}+1\right)\left\{r_{2}^{2}-\left[\left(a_{1}-4\right) m+7\right] r_{2}+6-\frac{6 m}{n}\right\}=0,
\end{aligned}
$$

for $\alpha_{1}=n$ and $\alpha_{2}=m$, respectively. Therefore $r_{j 0}=-1$ and $r_{j 1}=0, j=1,2$. In order to have distinct indices, if $r_{j 2} r_{j 3}=p_{j}$, then $p_{j}$, satisfy the following Diophantine equation

$$
\frac{1}{p_{1}}+\frac{1}{p_{2}}=\frac{1}{6}
$$

Among the solutions of the Diophantine equation (1.15), $\left(p_{1}, p_{2}\right)=(3,-6),(4,-12),(5,-30)$, $(8,24)$ and $(12,12)$ are the only ones lead to distinct Fuchs indices (resonances).

When $\left(p_{1}, p_{2}\right)=(3,-6)$, the distinct integer resonances for the first branch are $\left(r_{12}, r_{13}\right)=$ $(1,3)$. Then (1.14.a) implies that

$$
r_{12}+r_{13}=7+\left(a_{1}+4\right) n=4, \quad r_{12} r_{13}=6\left(1-\frac{n}{m}\right)=3 .
$$

The equations (1.16) give

$$
a_{1}=4-\frac{3}{n}, \quad m=2 n .
$$

respectively. (1.14.b) implies that, $r_{22}+r_{23}=1$ and $r_{22} r_{23}=-6$, and hence the distinct integer resonances for the second branch are $\left(r_{22}, r_{23}\right)=(-2,3)$. By using the equations (1.12.a) and (1.13), one obtains the following case

$$
\text { 4. }\left(a_{1}, a_{2}, a_{3}, a_{4}\right)=\left(4-\frac{3}{n}, 3-\frac{3}{n},-12+\frac{15}{n}-\frac{3}{n^{2}}, 6-\frac{9}{n}+\frac{3}{n^{2}}\right),
$$

with the resonances $\left(r_{11}, r_{12}, r_{13}\right)=(0,1,3)$ and $\left(r_{21}, r_{22}, r_{23}\right)=(0,-2,3)$.

Similarly, when $\left(p_{1}, p_{2}\right)=(4,-12)$, the resonances for the first and second branches respectively are $\left(r_{11}, r_{12}, r_{13}\right)=(0,1,4)$ and $\left(r_{21}, r_{22}, r_{23}\right)=(0,-3,4)$ and we have the following case

5. $\left(a_{1}, a_{2}, a_{3}, a_{4}\right)=\left(4-\frac{2}{n}, 3-\frac{4}{n},-12+\frac{14}{n}-\frac{2}{n^{2}}, 6-\frac{8}{n}+\frac{2}{n^{2}}\right)$,

When $\left(p_{1}, p_{2}\right)=(5,-30)$, by using the similar procedure, one obtains the following case

$$
\text { 6. }\left(a_{1}, a_{2}, a_{3}, a_{4}\right)=\left(4-\frac{1}{n}, 3-\frac{5}{n},-12+\frac{13}{n}-\frac{1}{n^{2}}, 6-\frac{7}{n}+\frac{1}{n^{2}}\right),
$$

with the resonances $\left(r_{11}, r_{12}, r_{13}\right)=(0,1,5)$ and $\left(r_{21}, r_{22}, r_{23}\right)=(0,-5,6)$.

When $\left(p_{1}, p_{2}\right)=(8,24)$, distinct integer resonances for the first branch are $\left(r_{12}, r_{13}\right)=$ $(1,8),(2,4)$, and $(-2,-4)$, but only the resonances $(2,4)$ leads to distinct integer resonances for the second branch. Hence, for this case the resonances are $\left(r_{11}, r_{12}, r_{13}\right)=(0,2,4)$, $\left(r_{21}, r_{22}, r_{23}\right)=(0,4,6)$, and

$$
\text { 7. }\left(a_{1}, a_{2}, a_{3}, a_{4}\right)=\left(4-\frac{1}{n}, 3-\frac{2}{n},-12+\frac{7}{n}+\frac{2}{n^{2}}, 6-\frac{4}{n}-\frac{2}{n^{2}}\right),
$$


For $\left(p_{1}, p_{2}\right)=(12,12)$, both branches have distinct integer resonances when the resonances for the first branch are $\left(r_{12}, r_{13}\right)=(3,4)$. Then the coefficients $a_{j}, j=1, \ldots, 4$ and the resonances are as follows:

$$
\text { 8. }\left(a_{1}, a_{2}, a_{3}, a_{4}\right)=\left(4,3,-12+\frac{6}{n^{2}}, 6-\frac{6}{n^{2}}\right),
$$

$\left(r_{j 1}, r_{j 2}, r_{j 3}\right)=(0,3,4), j=1,2$.

In the case of three branches, i.e. $a_{1}+a_{2}+a_{3}+a_{4} \neq 1$, let the roots of (1.4) be $\alpha_{1}=n, \alpha_{2}=m$ and $\alpha_{3}=l$ such that $\alpha_{i} \neq \alpha_{j}, i \neq j$, and $n, m, l \in \mathbb{Z}-\{0\}$. Then the equation (1.4) implies that

$$
\begin{gathered}
a_{1}+a_{2}+a_{3}+a_{4}-1=\frac{6}{n m l}, \quad 11-2 a_{1}-a_{2}=6\left(\frac{1}{n}+\frac{1}{m}+\frac{l}{m}\right), \\
6-3 a_{1}-2 a_{2}-a_{3}=6\left(\frac{1}{n l}+\frac{1}{m l}+\frac{1}{n m}\right) .
\end{gathered}
$$

Substituting (1.6) into (1.3), we obtain the following equations for the Fuchs indices $r_{j i}, j=$ $1,2,3, i=0,1,2,3$

$$
\begin{aligned}
& r_{1}\left(r_{1}+1\right)\left\{r_{1}^{2}-\left[\left(a_{1}-4\right) n+7\right] r_{1}+6\left(1-\frac{n}{m}\right)\left(1-\frac{n}{l}\right)\right\}=0, \\
& r_{2}\left(r_{2}+1\right)\left\{r_{2}^{2}-\left[\left(a_{1}-4\right) m+7\right] r_{2}+6\left(1-\frac{m}{n}\right)\left(1-\frac{m}{l}\right)\right\}=0, \\
& r_{3}\left(r_{3}+1\right)\left\{r_{3}^{2}-\left[\left(a_{1}-4\right) l+7\right] r_{3}+6\left(1-\frac{l}{m}\right)\left(1-\frac{l}{n}\right)\right\}=0,
\end{aligned}
$$

for $\alpha_{1}=n, \alpha_{2}=m$ and $\alpha_{3}=l$, respectively. Therefore, first two resonances for all three branches are $r_{j 0}=-1$ and $r_{j 1}=0, j=1,2,3$. If we let $p_{j}=r_{j 2} r_{j 3}$, then the equations (1.24) imply that

$$
p_{1}=6\left(1-\frac{n}{m}\right)\left(1-\frac{n}{l}\right), \quad p_{2}=6\left(1-\frac{m}{n}\right)\left(1-\frac{m}{l}\right), \quad p_{3}=6\left(1-\frac{l}{m}\right)\left(1-\frac{l}{n}\right),
$$

and hence $p_{j}$ satisfy the following Diophantine equation

$$
\sum_{j=1}^{3} \frac{1}{p_{j}}=\frac{1}{6} .
$$

Equation (1.26) implies that at least one of $p_{j}$ is positive integer. Let $p_{1}>0$, since

$$
p_{1} p_{2} p_{3}=-6^{3} \frac{(m-n)^{2}(l-n)^{2}(l-m)^{2}}{n^{2} m^{2} l^{2}},
$$

if we let $p_{2}>0$, then $p_{3}<0$. Among the solutions satisfying the condition $p_{1}, p_{2}>0$, and $p_{3}<0$ of the Diophantine equation $(1.26),\left(p_{1}, p_{2}, p_{3}\right)=(6, N,-N)$ where $N \in \mathbb{Z}_{+}$is the only one leads to distinct integer resonances for all three branches. If we let

$$
\lambda=6 \frac{(m-n)(l-n)(l-m)}{n m l},
$$

then equations (1.25) respectively give

$$
\lambda n-p_{1} m+p_{1} l=0, \quad p_{2} n+\lambda m-p_{2} l=0, \quad-p_{3} n+p_{3} m+\lambda l=0,
$$


and thus

$$
\lambda^{2}=-\left(p_{1} p_{2}+p_{2} p_{3}+p_{1} p_{3}\right) .
$$

Therefore, when $\left(p_{1}, p_{2}, p_{3}\right)=(6, N,-N), \lambda= \pm N$. For $\lambda=N,(1.29)$ implies that $n=0$ and $m=l$. Similarly for $\lambda=-N$,

$$
m=\frac{(6-N) n}{12}, \quad l=\frac{(6+N) n}{12},
$$

provided that $N \neq 6$, and $(6 \pm N) n / 12$ are integers. Since $p_{1}=6$, then the possible integer resonances for the first branch are $\left(r_{12}, r_{13}\right)=(1,6),(-2,-3)$ and $(2,3)$. When $\left(r_{12}, r_{13}\right)=$ $(1,6)$, there are no integer resonances for the second and third branches. Therefore, we have the following two subcases: When $\left(r_{12}, r_{13}\right)=(-2,-3)$, the equation (1.24.a) gives

$$
r_{12}+r_{13}=\left(a_{1}-4\right) n+7=5 .
$$

Thus, $a_{1}=4-12 / n$. Similarly, (1.24.b) and (1.24.c) respectively imply

$$
\begin{array}{ll}
r_{22}+r_{23}=1+N, & r_{22} r_{23}=N, \\
r_{32}+r_{33}=1-N, & r_{32} r_{33}=-N .
\end{array}
$$

Therefore, the resonances for the second and third branches are $\left(r_{22}, r_{23}\right)=(1, N)$ and $\left(r_{32}, r_{33}\right)=(1,-N)$, provided that $N \neq 1$. The coefficients $a_{2}, a_{3}$, and $a_{4}$ can be determined from the equations (1.23). Thus we have the following case:

$$
\text { 9. } \begin{aligned}
\left(a_{1}, a_{2}, a_{3}, a_{4}\right)=( & 4-\frac{12}{n}, 3-\frac{216+18 N^{2}}{\left(36-N^{2}\right) n},-12+\frac{1728}{\left(36-N^{2}\right) n}-\frac{1728}{\left(36-N^{2}\right) n^{2}}, \\
& \left.6+\frac{6 N^{2}-1080}{\left(36-N^{2}\right) n}+\frac{1728}{\left(36-N^{2}\right) n^{2}}-\frac{846}{\left(36-N^{2}\right) n^{3}}\right)
\end{aligned}
$$

such that $N \in \mathbb{Z}_{+}-\{1,6\}$, and $n, \frac{(6 \pm N) n}{12}$ are non-zero integers. It should be noted that, as $N \rightarrow \infty$ this case reduce to the third case given by (1.11).

When $\left(r_{12}, r_{13}\right)=(2,3)$, by following the similar procedure we obtain the following case:

$$
\text { 10. } \begin{aligned}
\left(a_{1}, a_{2}, a_{3}, a_{4}\right)= & \left(4-\frac{2}{n}, 3+\frac{2 k^{2}-26}{\left(1-k^{2}\right) n},-12+\frac{58-10 k^{2}}{\left(1-k^{2}\right) n}-\frac{48}{\left(1-k^{2}\right) n^{2}},\right. \\
& \left.6+\frac{6 k^{2}-30}{\left(1-k^{2}\right) n}+\frac{48}{\left(1-k^{2}\right) n^{2}}-\frac{24}{\left(1-k^{2}\right) n^{3}}\right)
\end{aligned}
$$

where $k=N / 6, k \in \mathbb{Z}_{+}-\{1,6\}$ and provided that $n, \frac{(1 \pm k) n}{2}$ are non-zero integers. It should be noted that, as $k \rightarrow \infty$ this case reduce to the second case given by (1.10).

Moreover, as $n \rightarrow \pm \infty$ we have the following case:

11. $\left(a_{1}, a_{2}, a_{3}, a_{4}\right)=(4,3,-12,6)$.

Thus, we have eleven cases, (1.9), (1.10), (1.11), (1.18), (1.19), (1.20), (1.21), (1.22), (1.34), (1.35) and (1.36) and all the corresponding equations pass the Painlevé test. Moreover, if one lets $u=\dot{y} / y,(1.3)$ yields the following third order polynomial type equations:

$$
\dddot{u}=\left(a_{1}-4\right) u \ddot{u}+\left(a_{2}-3\right) \dot{u}^{2}+\left(3 a_{1}+2 a_{2}+a_{3}-6\right) u^{2} \dot{u}+\left(a_{1}+a_{2}+a_{3}+a_{4}-1\right) u^{4} .
$$

For the case $11,(1.37)$ yields $\dddot{u}=0$, and for the cases 1-10 (1.37) yields a equation of Painlevé type with leading order $\alpha=-1$, and $u_{0}=n$, i.e. $u \cong n\left(t-t_{0}\right)^{-1}$ as $t \rightarrow t_{0}$. The equations in $u$ obtained form (1.37) for the cases 1-10 were examined by Chazy [13], Bureau [4] and in [6, 11].

In the following sections, the "simplified equations" that retain only the leading terms as $z \rightarrow z_{0}$ will be considered for $\alpha=-4,-3,-2$ and -1 with positive distinct resonances, and some simplified equations for $\alpha=-4,-3,-2$ with negative resonances. 


\section{Leading order $\alpha=-2$}

Equation (1.2) contains the leading terms for any $\alpha=n \in \mathbb{Z}-\{0\}$ as $z \rightarrow z_{0}$, if we do not take into account $F$. In this section, we consider the case $\alpha=-2$. By adding the terms of order -6 or greater as $z \rightarrow z_{0}$, we obtain the equation

$$
y^{(4)}=a_{1} \frac{y^{\prime} y^{\prime \prime \prime}}{y}+a_{2} \frac{\left(y^{\prime \prime}\right)^{2}}{y}+a_{3} \frac{\left(y^{\prime}\right)^{2} y^{\prime \prime}}{y^{2}}+a_{4} \frac{\left(y^{\prime}\right)^{4}}{y^{3}}+b_{1} y y^{\prime \prime}+b_{2}\left(y^{\prime}\right)^{2}+b_{3} y^{3}+F_{1}\left(y, y^{\prime}, y^{\prime \prime}, y^{\prime \prime \prime} ; z\right)
$$

where $b_{i}, i=1,2,3$ are constants and $F_{1}$ contains the terms of order -5 or greater as $z \rightarrow z_{0}$. We consider the case of $F_{1}=0$, i.e., the simplified equation for $\alpha=-2$.

Suppose that (1.9), (1.10), (1.11), (1.18), (1.19), (1.20), (1.21), (1.22), (1.34), (1.35) and (1.36) hold, and substitute $y \cong y_{0}\left(z-z_{0}\right)^{-2}+\beta\left(z-z_{0}\right)^{r-2}$, into (2.1) with $F_{1}=0$. Then we obtain the following equations for the Fuchs indices $r$ and $y_{0}$

$$
\begin{aligned}
Q(r)= & (r+1)\left[r^{3}+\left(2 a_{1}-15\right) r^{2}-\left(20 a_{1}+12 a_{2}+4 a_{3}+b_{1} y_{0}-86\right) r\right. \\
& +2\left[48 a_{1}+36 a_{2}+24 a_{3}+16 a_{4}+\left(3 b_{1}+2 b_{2}\right) y_{0}-120\right]=0, \\
b_{3} y_{0}^{2}+ & 2\left(3 b_{1}+2 b_{2}\right) y_{0}+4\left(12 a_{1}+9 a_{2}+6 a_{3}+4 a_{4}-30\right)=0,
\end{aligned}
$$

respectively [22]. (2.3) implies that, in general, there are two branches if $b_{3} \neq 0$. Now, we determine $y_{0 j}, j=1,2$ and $b_{i}$ for each cases of $\left(a_{1}, a_{2}, a_{3}, a_{4}\right)$ such that one branch is the principal branch, i.e. all the resonances are positive distinct integers (except $r_{0}=-1$ ). If $r_{0}=-1$ and $r_{j i}, i=1,2,3$ then (2.2) implies that

$$
\begin{aligned}
& \sum_{i=1}^{3} r_{j i}=-\left(2 a_{1}-15\right), \quad \sum_{\substack{i, k=1 \\
i \neq k}}^{3} r_{j i} r_{j k}=-\left(20 a_{1}+12 a_{2}+4 a_{3}+b_{1} y_{0 j}-86\right), \\
& \prod_{i=1}^{3} r_{j i}=-2\left[48 a_{1}+36 a_{2}+24 a_{3}+16 a_{4}+\left(3 b_{1}+2 b_{2}\right) y_{0 j}-120\right], \quad j=1,2
\end{aligned}
$$

provided that the right hand sides of (2.4) are positive integers for at least one of $y_{0 j}$. According to number of branches, the following cases should be considered separately.

Case I. $b_{3}=0$ : In this case, there is one branch.

For the case $1,\left(a_{1}, a_{2}, a_{3}, a_{4}\right)=\left(4,3-\frac{6}{n},-12+\frac{12}{n}, 6-\frac{6}{n}\right),(2.4)$ gives

$$
\sum_{i=1}^{3} r_{i}=7, \quad \sum_{\substack{i, j=1 \\ i \neq j}}^{3} r_{i} r_{j}=18+\frac{24}{n}-b_{1} y_{0}, \quad \prod_{i=1}^{3} r_{i}=12+\frac{24}{n} .
$$

In order to have a principal branch, $n$ takes the values of $\pm 1, \pm 2, \pm 3, \pm 4, \pm 6, \pm 8, \pm 12, \pm 24$. Among these values of $n$, only for $n=-6,-2$ there exists a principal branch with the resonances $\left(r_{1}, r_{2}, r_{3}\right)=(1,2,4)$ and $(0,1,6)$ respectively. Then, $b_{1}$ and $b_{2}$ can be determined from equations (2.5.b) and (2.3) respectively. For $n=-2,\left(b_{1}, b_{2}, b_{3}\right)=(0,0,0)$ that is, no additional leading term. For $n=-6$, we have the following simplified equation,

$$
y^{(4)}=4 \frac{y^{\prime} y^{\prime \prime \prime}}{y}+4 \frac{\left(y^{\prime \prime}\right)^{2}}{y}-14 \frac{\left(y^{\prime}\right)^{2} y^{\prime \prime}}{y^{2}}+7 \frac{\left(y^{\prime}\right)^{4}}{y^{3}}+2\left(y^{\prime}\right)^{2} .
$$

(2.6) does not pass the Painlevé test since the compatibility condition at $r_{2}=2$ is not satisfied identically. When $\alpha=-2$, in the case of the single branch for all cases (1.9), (1.10), (1.11), (1.18), (1.19), (1.20), (1.21), (1.22), (1.34), (1.35) and (1.36) there are no additional leading terms and the simplified equations are the same as the reduced equations (1.3) for $n=-2$. 
Case II. $b_{3} \neq 0$ : If $y_{0 j}, j=1,2, \quad\left(y_{01} \neq y_{02}\right)$, are the roots of $(2.3)$, and $\left(r_{j 1}, r_{j 2}, r_{j 3}\right)$ are the resonances corresponding to $y_{0 j}$, then let

$$
\prod_{i=1}^{3} r_{j i}=P\left(y_{0 j}\right)=p_{j} \quad j=1,2,
$$

where

$$
P\left(y_{0 j}\right)=2\left[120-48 a_{1}-36 a_{2}-24 a_{3}-16 a_{4}-\left(3 b_{1}+2 b_{2}\right) y_{0 j}\right], \quad j=1,2
$$

and $p_{j} \in \mathbb{Z}-\{0\}$. In order to have a principal branch, at least one of the $p_{j}$ should be a positive integer. (2.3) gives

$$
b_{3}=-\frac{2 q}{y_{01} y_{02}}, \quad 3 b_{1}+2 b_{2}=q\left(\frac{1}{y_{01}}+\frac{1}{y_{02}}\right) .
$$

where $q=60-24 a_{1}-18 a_{2}-12 a_{3}-8 a_{4}$. Then (2.8) can be written as

$$
p_{j}=2 q\left(1-\frac{y_{0 j}}{y_{0 k}}\right), \quad j, k=1,2, \quad j \neq k
$$

If $p_{1} p_{2} \neq 0$ and $q \neq 0$, then $p_{j}$ satisfy the following simple hyperbolic type of Diophantine equation

$$
\frac{1}{p_{1}}+\frac{1}{p_{2}}=\frac{1}{2 q}
$$

The general solution of (2.11) is given as

$$
p_{1}=2 q-d_{i}, \quad p_{2}=2 q\left(1-\frac{2 q}{d_{i}}\right)
$$

where $\left\{d_{i}\right\}$ is the set of divisors of $4 q^{2}$. For each cases (1.9), (1.10), (1.11), (1.18), (1.19), (1.20), (1.21), (1.22), (1.34), (1.35) and (1.36), from (2.4.a) one can find the possible resonances of the principal branch, then $p_{2}$ can be obtained from the Diophantine equation (2.11). Once $p_{2}$ is known, possible resonances for the second branch satisfying the conditions (2.10) and (2.4.c) can be determined. Then the coefficients $b_{1}$ and $b_{2}, b_{3}$ of the additional leading terms can be determined by using the equations (2.4.c) and (2.9) respectively.

For the double branch case, we have only the following equations which have the additional leading terms: For the case 4, (1.18);

$$
\begin{aligned}
& y^{(4)}=\frac{y^{\prime} y^{\prime \prime \prime}}{y}+7 y y^{\prime \prime}-3 y^{3}, \\
& y_{01}=2, \quad y_{02}=12, \quad\left(r_{11}, r_{12}, r_{13}\right)=(2,5,6), \quad\left(r_{21}, r_{22}, r_{23}\right)=(-5,6,12), \\
& y^{(4)}=3 \frac{y^{\prime} y^{\prime \prime \prime}}{y}+2 \frac{\left(y^{\prime \prime}\right)^{2}}{y}-\frac{22}{3} \frac{\left(y^{\prime}\right)^{2} y^{\prime \prime}}{y^{2}}+\frac{10}{3} \frac{\left(y^{\prime}\right)^{4}}{y^{3}}+8 y y^{\prime \prime}-\left(y^{\prime}\right)^{2}-6 y^{3}, \\
& y_{01}=2 / 3, \quad y_{02}=20 / 3, \quad\left(r_{11}, r_{12}, r_{13}\right)=(2,3,4), \quad\left(r_{21}, r_{22}, r_{23}\right)=(-5,6,8) .
\end{aligned}
$$

For the case 5, (1.19);

$$
\begin{aligned}
& y^{(4)}=3 \frac{y^{\prime} y^{\prime \prime \prime}}{y}+\frac{\left(y^{\prime \prime}\right)^{2}}{y}-\frac{11}{2} \frac{\left(y^{\prime}\right)^{2} y^{\prime \prime}}{y^{2}}+\frac{5}{2} \frac{\left(y^{\prime}\right)^{4}}{y^{3}}+5 y y^{\prime \prime}-\frac{5}{2}\left(y^{\prime}\right)^{2}-2 y^{3} \\
& y_{01}=2, \quad y_{02}=8, \quad\left(r_{11}, r_{12}, r_{13}\right)=(2,3,4), \quad\left(r_{21}, r_{22}, r_{23}\right)=(-3,4,8) .
\end{aligned}
$$


For the case 6, (1.20);

$$
\begin{aligned}
& y^{(4)}=3 \frac{y^{\prime} y^{\prime \prime \prime}}{y}-2 \frac{\left(y^{\prime \prime}\right)^{2}}{y}+24 y y^{\prime \prime}-18\left(y^{\prime}\right)^{2}-24 y^{3}, \\
& y_{01}=1, \quad y_{02}=2, \quad\left(r_{11}, r_{12}, r_{13}\right)=(2,3,4), \quad\left(r_{21}, r_{22}, r_{23}\right)=(-2,3,8) .
\end{aligned}
$$

For the case 8, (1.22);

$$
\begin{aligned}
& y^{(4)}=4 \frac{y^{\prime} y^{\prime \prime \prime}}{y}+3 \frac{\left(y^{\prime \prime}\right)^{2}}{y}-\frac{93}{8} \frac{\left(y^{\prime}\right)^{2} y^{\prime \prime}}{y^{2}}+\frac{45}{8} \frac{\left(y^{\prime}\right)^{4}}{y^{3}}+5 y y^{\prime \prime}-\frac{5}{2}\left(y^{\prime}\right)^{2}-4 y^{3}, \\
& y_{01}=1 / 2, \quad y_{02}=9 / 2, \quad \begin{array}{c}
\left(r_{11}, r_{12}, r_{13}\right)=(1,2,4), \quad\left(r_{21}, r_{22}, r_{23}\right)=(-3,4,6) .
\end{array}
\end{aligned}
$$

For the case 10, (1.35);

$$
\begin{aligned}
& y^{(4)}= 2 \frac{y^{\prime} y^{\prime \prime \prime}}{y}+\frac{3}{2} \frac{\left(y^{\prime \prime}\right)^{2}}{y}-2 \frac{\left(y^{\prime}\right)^{2} y^{\prime \prime}}{y^{2}}+18 y^{3}, \\
& y_{0 j}^{2}= 1, \quad\left(r_{j 1}, r_{j 2}, r_{j 3}\right)=(2,3,6), \quad j=1,2, \\
& y^{(4)}= 3 \frac{y^{\prime} y^{\prime \prime \prime}}{y}+\frac{5}{2} \frac{\left(y^{\prime \prime}\right)^{2}}{y}-\frac{15}{2} \frac{\left(y^{\prime}\right)^{2} y^{\prime \prime}}{y^{2}}+\frac{25}{8} \frac{\left(y^{\prime}\right)^{4}}{y^{3}}+6 y y^{\prime \prime}-4\left[\left(y^{\prime}\right)^{2}+y^{3}\right], \\
& y_{01}= 1, \quad y_{02}=4, \quad\left(r_{11}, r_{12}, r_{13}\right)=(1,2,6), \quad\left(r_{21}, r_{22}, r_{23}\right)=(-2,3,8), \\
& y^{(4)}= \frac{9}{2} \frac{y^{\prime} y^{\prime \prime \prime}}{y}+\left(\frac{7}{2}+\frac{6}{1-k^{2}}\right) \frac{\left(y^{\prime \prime}\right)^{2}}{y}-\left(\frac{29}{2}+\frac{15}{1-k^{2}}\right) \frac{\left(y^{\prime}\right)^{2} y^{\prime \prime}}{y^{2}} \\
&+\left(\frac{15}{2}+\frac{75}{1-k^{2}}\right) \frac{\left(y^{\prime}\right)^{4}}{y^{3}}+\left(k^{2}+11\right) y y^{\prime \prime}-15\left(y^{\prime}\right)^{2}+6\left(1-k^{2}\right) y^{3}, \\
& y_{01}=-1 /\left(1-k^{2}\right), \quad y_{02}=-k^{2} /\left(1-k^{2}\right), \\
&\left(r_{11}, r_{12}, r_{13}\right)=(1,2,3), \quad\left(r_{21}, r_{22}, r_{23}\right)=(6, k,-k), \quad k \neq 1,6 .
\end{aligned}
$$

For all the other cases, the simplified equations are the same as the reduced equations (1.3) with the coefficients for $n=-2$.

\section{$3 \quad$ Leading order $\alpha=-1$}

$\alpha=-1$ is also a possible leading order of the equation (1.2) if $F=0$. By adding terms of order -5 , we obtain the following simplified equation with the leading order $\alpha=-1$,

$$
\begin{aligned}
y^{(4)}= & a_{1} \frac{y^{\prime} y^{\prime \prime \prime}}{y}+a_{2} \frac{\left(y^{\prime \prime}\right)^{2}}{y_{3}}+a_{3} \frac{\left(y^{\prime}\right)^{2} y^{\prime \prime}}{y^{2}}+a_{4} \frac{\left(y^{\prime}\right)^{4}}{y^{3}}+c_{1} y y^{\prime \prime \prime}+c_{2} y^{\prime} y^{\prime \prime}+c_{3} \frac{\left(y^{\prime}\right)^{3}}{y}+c_{4} y^{2} y^{\prime \prime} \\
& +c_{5} y\left(y^{\prime}\right)^{2}+c_{6} y^{3} y^{\prime}+c_{7} y^{5},
\end{aligned}
$$

where $c_{k}, k=1, \ldots, 7$ are constants.

Suppose that (1.9), (1.10), (1.11), (1.18), (1.19), (1.20), (1.21), (1.22), (1.34), (1.35) and (1.36) hold. If we substitute $y \cong y_{0}\left(z-z_{0}\right)^{-1}+\beta\left(z-z_{0}\right)^{r-1}$ into (3.1), then we obtain the following equations for the Fuchs indices $r$ and $y_{0}$

$$
\begin{aligned}
Q(r)= & (r+1)\left\{r^{3}+\left(a_{1}-c_{1} y_{0}-11\right) r^{2}-\left[7 a_{1}+4 a_{2}+a_{3}-\left(7 c_{1}+c_{2}\right) y_{0}+c_{4} y_{0}^{2}-46\right] r\right. \\
& +24 a_{1}+16 a_{2}+8 a_{3}+4 a_{4}-96-3\left(6 c_{1}+2 c_{2}+c_{3}\right) y_{0} \\
& \left.+2\left(2 c_{4}+c_{5}\right) y_{0}^{2}-c_{6} y_{0}^{3}\right\}=0 \\
& c_{7} y_{0}^{4}-c_{6} y_{0}^{3}+\left(c_{5}+2 c_{4}\right) y_{0}^{2}-\left(c_{3}+2 d_{2}+6 c_{1}\right) y_{0}+6 a_{1}+4 a_{2}+2 a_{3}+a_{4}-24=0
\end{aligned}
$$


respectively. (3.3) implies that, in general, there are four branches if $c_{7} \neq 0$. Now, we determine $y_{0 j}, j=1, \ldots, 4$, and $c_{k}$ for each cases of $\left(a_{1}, a_{2}, a_{3}, a_{4}\right)$ such that one branch is the principal branch. If $r_{0}=-1$ and $r_{j i}, j=1, \ldots, 4, i=1,2,3$ then (3.2) implies that

$$
\begin{gathered}
\sum_{i=1}^{3} r_{j i}=11-a_{1}+c_{1} y_{0}, \quad \sum_{\substack{i, k=1 \\
i \neq k}}^{3} r_{j i} r_{j k}=46-7 a_{1}-4 a_{2}-a_{3}+\left(7 c_{1}+c_{2}\right) y_{0}-c_{4} y_{0}^{2}, \\
\prod_{i=1}^{3} r_{j i}=96-24 a_{1}-16 a_{2}-8 a_{3}-4 a_{4}+3\left(6 c_{1}+2 c_{2}+c_{3}\right) y_{0} \\
-2\left(2 c_{4}+c_{5}\right) y_{0}^{2}+c_{6} y_{0}^{3}, \quad j=1, \ldots, 4
\end{gathered}
$$

According to number of branches, the following cases should be considered separately.

Case I. $c_{4}=c_{5}=c_{6}=c_{7}=0$ : In this case, there is one branch. From (3.4.c) one can find the possible resonances of the single principal branch for each cases (1.9), (1.10), (1.11), (1.18), (1.19), (1.20), (1.21), (1.22), (1.34), (1.35) and (1.36). Then the coefficients $c_{1}, c_{2}$, and $c_{3}$ can be determined by using the equations (3.3), (3.4.a) and (3.4.b). For the cases (1.9), (1.10), (1.11), (1.18), (1.19), (1.20), (1.21) and (1.22), and for all possible values of $n$, there is no simplified equation which passes the Painlevé test. Hence, for these cases the simplified equations are the same as the reduced equations (1.3) with the coefficients for $n=-1$ For the cases (1.34) and (1.35), the right hand side of (3.4.c) depends on $N$ and $k$ respectively. When (1.34) holds, for $n=1, \pm 2, \pm 3, \pm 4, \pm 6, \pm 12$, and when (1.35) holds for $n=1, \pm 2$ the simplified equations do not pass the Painlevé test. Hence, for these cases and for these particular values of $n$, the simplified equations are the same as the reduced equation (1.3) with the coefficients for $n=-1$. For the case (1.36), we obtain the following simplified equation:

$$
\begin{aligned}
& y^{(4)}=4 \frac{y^{\prime} y^{\prime \prime \prime}}{y}+3 \frac{\left(y^{\prime \prime}\right)^{2}}{y}-12 \frac{\left(y^{\prime}\right)^{2} y^{\prime \prime}}{y^{2}}+6 \frac{\left(y^{\prime}\right)^{4}}{y^{3}}-y y^{\prime \prime \prime}, \\
& y_{01}=1, \quad\left(r_{1}, r_{2}, r_{3}\right)=(1,2,3) .
\end{aligned}
$$

Case II. $c_{6}=c_{7}=0$ : In this case, there are two branches. If $y_{0 j}, j=1,2,\left(y_{01} \neq y_{02}\right)$, are the roots of $(3.3)$, and $\left(r_{j 1}, r_{j 2}, r_{j 3}\right)$ are the resonances corresponding to $y_{0 j}$, then let

$$
\prod_{i=1}^{3} r_{j i}=P\left(y_{0 j}\right)=p_{j} \quad j=1,2,
$$

where

$$
P\left(y_{0 j}\right)=-\left[24 a_{1}+16 a_{2}+8 a_{3}+4 a_{4}-96-3\left(6 c_{1}+2 c_{2}+c_{3}\right) y_{0 j}+2\left(2 c_{4}+c_{5}\right) y_{0 j}^{2}\right], \quad j=1,2,
$$

and $p_{j} \in \mathbb{Z}-\{0\}$. In order to have a principal branch, at least one of the $p_{j}$ should be a positive integer. (3.3) gives

$$
c_{5}+2 c_{4}=-\frac{s}{y_{01} y_{02}}, \quad c_{3}+2 c_{2}+c_{1}=-s\left(\frac{1}{y_{01}}+\frac{1}{y_{02}}\right) .
$$

where

$$
s=24-6 a_{1}-4 a_{2}-2 a_{3}-a_{4} .
$$

Then (3.7) can be written as

$$
p_{j}=s\left(1-\frac{y_{0 j}}{y_{0 k}}\right), \quad j, k=1,2, \quad j \neq k
$$


If $\prod p_{j} \neq 0$ and $s \neq 0$, then $p_{j}$ satisfy the following simple hyperbolic type of Diophantine equation

$$
\frac{1}{p_{1}}+\frac{1}{p_{2}}=\frac{1}{s}
$$

The general solution of (2.11) is given as

$$
p_{1}=s-d_{i}, \quad p_{2}=s\left(1-\frac{s}{d_{i}}\right)
$$

where $\left\{d_{i}\right\}$ is the set of divisors of $s^{2}$. For each cases (1.9), (1.10), (1.11), (1.18), (1.19), (1.20), (1.21), (1.22), (1.34), (1.35) and (1.36), from (3.4.a) one can find the possible resonances of the principal branch, consequently $p_{1}$, then $p_{2}$ can be obtained from the Diophantine equation (3.11). Once $p_{2}$ is known, possible resonances for the second branch satisfying the conditions (3.4) can be determined. Then the coefficients $c_{k}$ of the additional leading terms can be determined by using the equations (3.4) and (3.8). One should consider the cases $c_{1}=0$ and $c_{1} \neq 0$ separately.

II.a. $c_{1}=0$ : For this case, we have the following equations which admit additional leading terms: For the case 2, (1.10);

$$
\begin{aligned}
& y^{(4)}=2 \frac{y^{\prime} y^{\prime \prime \prime}}{y}+\frac{\left(y^{\prime \prime}\right)^{2}}{y}-2 \frac{\left(y^{\prime}\right)^{2} y^{\prime \prime}}{y^{2}}+4\left[y^{2} y^{\prime \prime}+\left(y^{\prime}\right)^{2}\right], \\
& y_{0 j}^{2}=1, \quad\left(r_{j 1}, r_{j 2}, r_{j 3}\right)=(2,3,4) \quad j=1,2 .
\end{aligned}
$$

For the case 3, (1.11);

$$
\begin{aligned}
& y^{(4)}=5 \frac{y^{\prime} y^{\prime \prime \prime}}{y}+\frac{3}{2} \frac{\left(y^{\prime \prime}\right)^{2}}{y}-12 \frac{\left(y^{\prime}\right)^{2} y^{\prime \prime}}{y^{2}}+\frac{13}{2} \frac{\left(y^{\prime}\right)^{4}}{y^{3}}-5\left[y^{\prime} y^{\prime \prime}-\frac{\left(y^{\prime}\right)^{3}}{y}\right]+y^{2} y^{\prime \prime}-\frac{15}{2} y\left(y^{\prime}\right)^{2}, \\
& y_{01}=1, \quad y_{02}=-11, \quad\left(r_{11}, r_{12}, r_{13}\right)=(1,2,3), \quad\left(r_{21}, r_{22}, r_{23}\right)=(-2,-3,11) .
\end{aligned}
$$

For all the other cases, the simplified equations are the same as the reduced equations (1.3) with the coefficients for $n=-1$.

II.b. $c_{1} \neq 0$ : When $n=1$, we obtain the following equations which admit the additional leading terms: For the case 1, (1.9);

$$
\begin{aligned}
& y^{(4)}=4 \frac{y^{\prime} y^{\prime \prime \prime}}{y}-3 \frac{\left(y^{\prime \prime}\right)^{2}}{y}-y y^{\prime \prime \prime}-6\left[2 y^{\prime} y^{\prime \prime}-2 \frac{\left(y^{\prime}\right)^{3}}{y}+y\left(y^{\prime}\right)^{2}\right], \\
& y_{01}=1, \quad y_{02}=2, \quad\left(r_{11}, r_{12}, r_{13}\right)=(1,2,3), \quad\left(r_{21}, r_{22}, r_{23}\right)=(-2,1,6) .
\end{aligned}
$$

For the case 2, (1.10);

$$
\begin{aligned}
& y^{(4)}=2 \frac{y^{\prime} y^{\prime \prime \prime}}{y}+\frac{\left(y^{\prime \prime}\right)^{2}}{y}-2 \frac{\left(y^{\prime}\right)^{2} y^{\prime \prime}}{y^{2}}-3 y y^{\prime \prime \prime}-2\left[y^{2} y^{\prime \prime}+y\left(y^{\prime}\right)^{2}\right], \\
& y_{01}=1, \quad y_{02}=2, \quad\left(r_{11}, r_{12}, r_{13}\right)=(1,2,3), \quad\left(r_{21}, r_{22}, r_{23}\right)=(-2,2,3) .
\end{aligned}
$$

For all the other cases, the simplified equations are the same as the reduced equations (1.3) with the coefficients for $n=-1$.

Case III. $c_{7}=0$ : In this case, there are three branches. If $y_{0 j}, j=1,2,3\left(y_{0 j} \neq y_{0 k}, j \neq k\right)$, are the roots of $(3.3)$, and $\left(r_{j 1}, r_{j 2}, r_{j 3}\right)$ are the resonances corresponding to $y_{0 j}$, then let

$$
\prod_{i=1}^{3} r_{j i}=P\left(y_{0 j}\right)=p_{j} \quad j=1,2,3
$$


where

$$
P\left(y_{0 j}\right)=-\left[24 a_{1}+16 a_{2}+8 a_{3}+4 a_{4}-96-3\left(6 c_{1}+2 c_{2}+c_{3}\right) y_{0 j}+2\left(2 c_{4}+c_{5}\right) y_{0 j}^{2}-c_{6} y_{0 j}^{3}\right], \quad j=1,2,3,
$$

and $p_{j} \in \mathbb{Z}-\{0\}$. In order to have a principal branch, at least one of the $p_{j}$ should be a positive integer. (3.3) gives

$$
\begin{gathered}
c_{6}=-s \prod_{j=1}^{3} \frac{1}{y_{0 j}}, \quad c_{5}+2 c_{4}=-s \prod_{j=1}^{3} \frac{1}{y_{0 j}}\left(\sum_{j=1}^{3} y_{0 j}\right), \\
c_{3}+2 c_{2}+6 c_{1}=-s \prod_{j=1}^{3} \frac{1}{y_{0 j}}\left(\sum_{\substack{j, k=1, j \neq k}}^{3} y_{0 j} y_{0 k}\right)
\end{gathered}
$$

where $s$ is given in (3.9). Then (3.18) can be written as

$$
p_{j}=s \prod_{\substack{k=1 \\ k \neq j}}^{3}\left(1-\frac{y_{0 j}}{y_{0 k}}\right), \quad j=1, \ldots, 4,
$$

If $\prod p_{j} \neq 0$ and $s \neq 0$, then $p_{j}$ satisfy the following Diophantine equation

$$
\sum_{j=1}^{3} \frac{1}{p_{j}}=\frac{1}{s} .
$$

One should consider the cases $c_{1}=0$ and $c_{1} \neq 0$ separately.

III.a. $c_{1}=0$ : For each cases (1.9), (1.10), (1.11), (1.18), (1.19), (1.20), (1.21), (1.22), (1.34), (1.35) and (1.36), from (3.4.a) one can find the possible resonances of the principal branch, consequently $p_{1}$, then the Diophantine equation (3.21) can be reduced to the following simple hyperbolic type which can be solved in closed form:

$$
\frac{1}{p_{2}}+\frac{1}{p_{3}}=\frac{k_{1}}{k_{2}}
$$

where $k_{1}=p_{1}-s$ and $k_{2}=s p_{1}$. The general solution of (3.22) is given as

$$
p_{2}=\frac{k_{2}+d_{i}}{k_{1}}, \quad p_{3}=\frac{k_{2}\left(k_{2}+d_{i}\right)}{k_{1} d_{i}}
$$

where $\left\{d_{i}\right\}$ is the set of divisors of $k_{2}^{2}$. For the cases (1.10), (1.11), (1.18), (1.19), (1.20), (1.21) and (1.36), and for all $n \neq-1$ there is no simplified equation which passes the Painlevé test. For the case (1.9), (1.22), (1.34) and (1.35) when $n=1, \pm 2, \pm 3, n^{2}=4,9, n=$ $1, \pm 2, \pm 3, \pm 4, \pm 6, \pm 12, N=2,3$ and $n=1,2,-2, k=2,3,4$ respectively, there is no simplified equation which passes the Painlevé test.

III.b. $c_{1} \neq 0$ : Equation (3.20) implies that

$$
\prod_{j=1}^{3} p_{j}=-s^{3} \frac{\left(y_{01}-y_{02}\right)^{2}\left(y_{02}-y_{03}\right)^{2}\left(y_{03}-y_{01}\right)^{2}}{y_{01}^{2} y_{02}^{2} y_{03}^{2}} .
$$

Hence, if $s>0$, let $p_{1}>0$ then $p_{2}>0, p_{3}<0$, and if $s<0$, let $p_{1}>0$ then $p_{2}, p_{3}<0$. For $s>0$, suppose that $p_{1}<p_{2}$, then from (3.21) one obtains $p_{1}<2 s$. Similarly, for $s<0$, one 
obtains $p_{1}<s$. Since for both cases $s>0$ and $s<0, p_{1}>0$ and bounded by $2 s$ and $s$ from above respectively, the Diophantine equation (3.21) can be reduced to a simple hyperbolic type in $p_{2}$ and $p_{3}$ which has the closed form solution given by (3.23). Once $p_{2}$ and $p_{3}$ are known, the possible resonances for the second and third branches satisfying the conditions (3.4) can be determined. Then the coefficients $c_{k}$ of the additional leading terms can be determined by using the equations (3.4) and (3.19).

For $n=2$ (for the case 9, (1.34): $n=2, N=18,24$, and for the case 10, $(1.35): n=2, k=$ $3,4)$, we have the following simplified equations which admit the additional leading terms: For the case $4,(1.18)$;

$$
\begin{aligned}
y^{(4)}= & \frac{5}{2} \frac{y^{\prime} y^{\prime \prime \prime}}{y}+\frac{3}{2} \frac{\left(y^{\prime \prime}\right)^{2}}{y}-\frac{21}{4} \frac{\left(y^{\prime}\right)^{2} y^{\prime \prime}}{y^{2}}+\frac{9}{4} \frac{\left(y^{\prime}\right)^{4}}{y^{3}}+y y^{\prime \prime \prime}+\frac{3}{5} y^{\prime} y^{\prime \prime}-\frac{3}{10} \frac{\left(y^{\prime}\right)^{3}}{y} \\
& -\frac{9}{4} y\left[y y^{\prime \prime}+\left(y^{\prime}\right)^{2}\right]+\frac{6}{125} y^{3} y^{\prime},
\end{aligned}
$$

$y_{01}=-5 / 2, \quad y_{02}=-25 / 2, \quad y_{03}=-15 / 2$,

$\left(r_{11}, r_{12}, r_{13}\right)=(1,2,3), \quad\left(r_{21}, r_{22}, r_{23}\right)=(-5,-2,3), \quad\left(r_{31}, r_{32}, r_{33}\right)=(-3,1,3)$.

For the case 5, (1.19);

$$
\begin{aligned}
& y^{(4)}=3 \frac{y^{\prime} y^{\prime \prime \prime}}{y}+\frac{\left(y^{\prime \prime}\right)^{2}}{y}-\frac{11}{2} \frac{\left(y^{\prime}\right)^{2} y^{\prime \prime}}{y^{2}}+\frac{5}{2} \frac{\left(y^{\prime}\right)^{4}}{y^{3}}+y y^{\prime \prime \prime}+\frac{3}{2} y^{\prime} y^{\prime \prime}-\frac{5}{4} \frac{\left(y^{\prime}\right)^{3}}{y} \\
& \quad-\frac{1}{8} y\left[3 y y^{\prime \prime}+5\left(y^{\prime}\right)^{2}\right]+\frac{1}{16} y^{3} y^{\prime}, \\
& y_{01}=-2, \quad y_{02}=-14, \quad y_{03}=-6, \\
& \left(r_{11}, r_{12}, r_{13}\right)=(1,2,3), \quad\left(r_{21}, r_{22}, r_{23}\right)=(-7,-3,4), \quad\left(r_{31}, r_{32}, r_{33}\right)=(-3,1,4) .
\end{aligned}
$$

For the case $6,(1.20)$;

$$
\begin{aligned}
& y^{(4)}= \frac{7}{2} \frac{y^{\prime} y^{\prime \prime \prime}}{y}+\frac{1}{2} \frac{\left(y^{\prime \prime}\right)^{2}}{y}-\frac{23}{4} \frac{\left(y^{\prime}\right)^{2} y^{\prime \prime}}{y^{2}}+\frac{11}{4} \frac{\left(y^{\prime}\right)^{4}}{y^{3}}+y y^{\prime \prime \prime}+3 y^{\prime} y^{\prime \prime}-\frac{17}{6} \frac{\left(y^{\prime}\right)^{3}}{y} \\
&-\frac{1}{3} y\left[y y^{\prime \prime}+\frac{11}{3}\left(y^{\prime}\right)^{2}-\frac{2}{9} y^{2} y^{\prime}\right], \\
& y_{01}=-3 / 2, \quad y_{02}=-39 / 2, \quad y_{03}=-9 / 2, \\
&\left(r_{11}, r_{12}, r_{13}\right)=(1,2,3), \quad\left(r_{21}, r_{22}, r_{23}\right)=(-13,-5,6), \quad\left(r_{31}, r_{32}, r_{33}\right)=(-3,1,5) .
\end{aligned}
$$

For the case $7,(1.21)$;

$$
\begin{aligned}
& y^{(4)}=\frac{7}{2} \frac{y^{\prime} y^{\prime \prime \prime}}{y}+2 \frac{\left(y^{\prime \prime}\right)^{2}}{y}-8 \frac{\left(y^{\prime}\right)^{2} y^{\prime \prime}}{y^{2}}+\frac{7}{2} \frac{\left(y^{\prime}\right)^{4}}{y^{3}}+y y^{\prime \prime \prime}-\frac{1}{3} \frac{\left(y^{\prime}\right)^{3}}{y}-\frac{2}{9} y\left[\left(y^{\prime}\right)^{2}+\frac{2}{3} y^{2} y^{\prime}\right], \\
& y_{01}=-3 / 2, \quad y_{02}=15 / 2, \quad y_{03}=-9 / 2, \\
& \left(r_{11}, r_{12}, r_{13}\right)=(1,2,3), \quad\left(r_{21}, r_{22}, r_{23}\right)=(4,5,6), \quad\left(r_{31}, r_{32}, r_{33}\right)=(-3,2,4) .
\end{aligned}
$$

For the case 8, (1.22);

$$
\begin{aligned}
& y^{(4)}=4 \frac{y^{\prime} y^{\prime \prime \prime}}{y}+3 \frac{\left(y^{\prime \prime}\right)^{2}}{y}-\frac{21}{2} \frac{\left(y^{\prime}\right)^{2} y^{\prime \prime}}{y^{2}}+\frac{9}{2} \frac{\left(y^{\prime}\right)^{4}}{y^{3}}+y y^{\prime \prime \prime}-3 y^{\prime} y^{\prime \prime}+\frac{3}{2} \frac{\left(y^{\prime}\right)^{3}}{y} \\
& \quad+\frac{3}{2} y\left[y y^{\prime \prime}+\left(y^{\prime}\right)^{2}\right]-\frac{3}{2} y^{3} y^{\prime}, \\
& y_{01}=-1, \quad y_{02}=1, \quad y_{03}=-3, \\
& \left(r_{11}, r_{12}, r_{13}\right)=(1,2,3), \quad\left(r_{21}, r_{22}, r_{23}\right)=(1,3,4), \quad\left(r_{31}, r_{32}, r_{33}\right)=(-3,3,4) .
\end{aligned}
$$

Case IV. $c_{7} \neq 0$ : In this case, there are four branches. If $y_{0 j}, j=1, \ldots, 4\left(y_{0 j} \neq y_{0 k}, j \neq k\right)$, are the roots of (3.3), and $\left(r_{j 1}, r_{j 2}, r_{j 3}\right)$ are the resonances corresponding to $y_{0 j}$, then let

$$
\prod_{i=1}^{3} r_{j i}=P\left(y_{0 j}\right)=p_{j} \quad j=1, \ldots, 4,
$$


where

$$
P\left(y_{0 j}\right)=-\left[24 a_{1}+16 a_{2}+8 a_{3}+4 a_{4}-96-3\left(6 c_{1}+2 c_{2}+c_{3}\right) y_{0 j}+2\left(2 c_{4}+c_{5}\right) y_{0 j}^{2}-c_{6} y_{0 j}^{3}\right], \quad j=1, \ldots, 4,
$$

and $p_{j} \in \mathbb{Z}-\{0\}$. In order to have a principal branch, at least one of the $p_{j}$ should be a positive integer. When $c_{1}=0,(3.3)$ gives

$$
\begin{aligned}
& c_{7}=-s \prod_{j=1}^{4} \frac{1}{y_{0 j}}, \quad c_{6}=-s \prod_{j=1}^{4} \frac{1}{y_{0 j}}\left(\sum_{j=1}^{4} y_{0 j}\right), \\
& c_{5}+2 c_{4}=-s \prod_{j=1}^{4} \frac{1}{y_{0 j}}\left(\sum_{\substack{j, k=1, j \neq k}}^{4} y_{0 j} y_{0 k}\right), \quad c_{3}+2 c_{2}=-s \prod_{j=1}^{4} \frac{1}{y_{0 j}}\left(\sum_{\substack{j, k, l=1, j \neq k \neq l}}^{4} y_{0 j} y_{0 k} y_{0 l}\right),
\end{aligned}
$$

where $s$ is given in (3.9). Then (3.31) can be written as

$$
p_{j}=s \prod_{\substack{k=1 \\ k \neq j}}^{4}\left(1-\frac{y_{0 j}}{y_{0 k}}\right), \quad j=1, \ldots, 4,
$$

If $\prod p_{j} \neq 0$ and $s \neq 0$, then $p_{j}$ satisfy the following Diophantine equation

$$
\sum_{j=1}^{4} \frac{1}{p_{j}}=\frac{1}{s} .
$$

If we let $p_{1}=p_{2}$ and $p_{3}=p_{4}$, then (3.34) can be reduced to simple hyperbolic type of Diophantine equation which admits closed form solution (3.12). When $p_{1}=p_{2}$ and $p_{3}=p_{4}$, such that $p_{1}>0$, for each cases (1.9), (1.10), (1.11), (1.18), (1.19), (1.20), (1.21), (1.22), (1.34), (1.35) and (1.36), from (3.4.a) one can find the possible resonances for the branches satisfying the conditions (3.4). Then the coefficients $c_{k}, k=2, \ldots, 7$ of the additional leading terms can be determined by using the equations (3.4) and (3.32).

For this particular case, we have the following equations which admit additional leading terms: For the case 5, (1.19);

$$
\begin{aligned}
& y^{(4)}=2 \frac{y^{\prime} y^{\prime \prime \prime}}{y}-\frac{\left(y^{\prime \prime}\right)^{2}}{y}+5 y^{2} y^{\prime \prime}-y^{5}, \\
& y_{01}^{2}=2, \quad y_{02}=-y_{01}, \quad y_{03}^{2}=8, \quad y_{04}=-y_{03} \\
& \left(r_{j 1}, r_{j 2}, r_{j 3}\right)=(2,3,4), \quad j=1,2, \quad\left(r_{j 1}, r_{j 2}, r_{j 3}\right)=(-3,4,8), \quad j=3,4 .
\end{aligned}
$$

For the case $7,(1.21)$;

$$
\begin{aligned}
& y^{(4)}=3 \frac{y^{\prime} y^{\prime \prime \prime}}{y}+\frac{\left(y^{\prime \prime}\right)^{2}}{y}-3 \frac{\left(y^{\prime}\right)^{2} y^{\prime \prime}}{y^{2}}+5 y^{2} y^{\prime \prime}-2 y^{5}, \\
& y_{01}^{2}=1, \quad y_{02}=-y_{01}, \quad y_{03}^{2}=4, \quad y_{04}=-y_{03} \\
& \left(r_{j 1}, r_{j 2}, r_{j 3}\right)=(1,3,4), \quad j=1,2, \quad\left(r_{j 1}, r_{j 2}, r_{j 3}\right)=(-2,4,6), \quad j=3,4 .
\end{aligned}
$$

For the case $8,(1.22)$;

$$
\begin{aligned}
& y^{(4)}=4 \frac{y^{\prime} y^{\prime \prime \prime}}{y}+3 \frac{\left(y^{\prime \prime}\right)^{2}}{y}-\frac{21}{2} \frac{\left(y^{\prime}\right)^{2} y^{\prime \prime}}{y^{2}}+\frac{9}{2} \frac{\left(y^{\prime}\right)^{4}}{y^{3}}+\frac{5}{2} y^{2} y^{\prime \prime}-\frac{1}{2} y^{5}, \\
& y_{01}^{2}=1, \quad y_{02}=-y_{01}, \quad y_{03}^{2}=9, \quad y_{04}=-y_{03} \\
& \left(r_{j 1}, r_{j 2}, r_{j 3}\right)=(1,2,4), \quad j=1,2, \quad\left(r_{j 1}, r_{j 2}, r_{j 3}\right)=(-3,4,6), \quad j=3,4 .
\end{aligned}
$$


If one lets $y=1 / u$, then (3.37) gives

$$
u^{(4)}=4 \frac{u^{\prime} u^{\prime \prime \prime}}{u}+3 \frac{\left(u^{\prime \prime}\right)^{2}}{u}-\frac{21}{2} \frac{\left(u^{\prime}\right)^{2} u^{\prime \prime}}{u^{2}}+\frac{9}{2} \frac{\left(u^{\prime}\right)^{4}}{u^{3}}+5 \frac{u^{\prime \prime}}{u^{3}}-10 \frac{\left(u^{\prime}\right)^{2}}{u^{3}}+\frac{2}{u^{3}} .
$$

The canonical form (equation also contains the terms of order -4 or greater as $z \rightarrow z_{0}$ ) of (3.38) was also given in $[18,20]$.

For the case 10, (1.35);

$$
\begin{aligned}
& y^{(4)}=2 \frac{y^{\prime} y^{\prime \prime \prime}}{y}+2 \frac{\left(y^{\prime \prime}\right)^{2}}{y}-2 \frac{\left(y^{\prime}\right)^{2} y^{\prime \prime}}{y^{2}}+6 y^{2} y^{\prime \prime}-2 y\left(y^{\prime}\right)^{2}-2 y^{5}, \\
& y_{01}^{2}=1, \quad y_{02}=-y_{01}, \quad 4, \quad y_{04}=-y_{03} \\
& \left(r_{j 1}, r_{j 2}, r_{j 3}\right)=(1,2,6), \quad j=1,2, \quad\left(r_{j 1}, r_{j 2}, r_{j 3}\right)=(-2,3,8), \quad j=3,4 . \\
& y^{(4)}=2 \frac{y^{\prime} y^{\prime \prime \prime}}{y}+2 \frac{\left(y^{\prime \prime}\right)^{2}}{y}-2 \frac{\left(y^{\prime}\right)^{2} y^{\prime \prime}}{y^{2}}+3 y^{2} y^{\prime \prime}+\frac{5}{2} y\left(y^{\prime}\right)^{2}-\frac{1}{2} y^{5}, \\
& y_{01}^{2}=1, \quad y_{02}=-y_{01}, \quad y_{04}=-y_{03} \\
& \left(r_{j 1}, r_{j 2}, r_{j 3}\right)=(1,3,5), \quad j=1,2, \quad\left(r_{j 1}, r_{j 2}, r_{j 3}\right)=(-5,6,8), \quad j=3,4 . \\
& y^{(4)}=2 \frac{y^{\prime} y^{\prime \prime \prime}}{y}+\frac{3}{2} \frac{\left(y^{\prime \prime}\right)^{2}}{y}-2 \frac{\left(y^{\prime}\right)^{2} y^{\prime \prime}}{y^{2}}+5 y^{2} y^{\prime \prime}+\frac{5}{2} y\left(y^{\prime}\right)^{2}-\frac{5}{2} y^{5}, \\
& y_{01}^{2}=1, \quad y_{02}=-y_{01}, \quad y_{04}=-y_{03} \\
& \left(r_{j 1}, r_{j 2}, r_{j 3}\right)=(1,3,5), \quad j=1,2, \quad\left(r_{j 1}, r_{j 2}, r_{j 3}\right)=(-2,5,6), \quad j=3,4 .
\end{aligned}
$$

The canonical form of (3.41) was given in [18, 19, 20, 21].

$$
\begin{aligned}
& y^{(4)}=3 \frac{y^{\prime} y^{\prime \prime \prime}}{y}+\frac{7}{2} \frac{\left(y^{\prime \prime}\right)^{2}}{y}-\frac{17}{2} \frac{\left(y^{\prime}\right)^{2} y^{\prime \prime}}{y^{2}}+\frac{27}{8} \frac{\left(y^{\prime}\right)^{4}}{y^{3}}+5 y^{2} y^{\prime \prime}+\frac{5}{2}\left[y\left(y^{\prime}\right)^{2}-y^{5}\right], \\
& y_{01}^{2}=1 / 2, \quad y_{02}=-y_{01}, \quad y_{03}^{2}=9 / 2, \quad y_{04}=-y_{03} \\
& \left(r_{j 1}, r_{j 2}, r_{j 3}\right)=(1,2,5), \quad j=1,2, \quad\left(r_{j 1}, r_{j 2}, r_{j 3}\right)=(-3,5,6), \quad j=3,4, \\
& y^{(4)}=5 \frac{y^{\prime} y^{\prime \prime \prime}}{y}+\frac{4\left(4-k^{2}\right)}{1-k^{2}} \frac{\left(y^{\prime \prime}\right)^{2}}{y}-\frac{53-17 k^{2}\left(y^{\prime}\right)^{2} y^{\prime \prime}}{1-k^{2}}+\frac{9\left(4-k^{2}\right)}{y^{2}} \frac{\left(y^{\prime}\right)^{4}}{y^{3}} \\
& \quad+\left(k^{2}+11\right) y^{2} y^{\prime \prime}+\left(k^{2}-19\right) y\left(y^{\prime}\right)^{2}+3\left(1-k^{2}\right) y^{5}, \\
& y_{01}^{2}=1 /\left(k^{2}-1\right), \quad y_{02}=-y_{01}, \quad y_{03}^{2}=k^{2} /\left(k^{2}-1\right), \quad y_{04}=-y_{03} \\
& \left(r_{j 1}, r_{j 2}, r_{j 3}\right)=(1,2,3), \quad j=1,2, \quad\left(r_{j 1}, r_{j 2}, r_{j 3}\right)=(6, k,-k), \quad j=3,4 .
\end{aligned}
$$

When $k=2,(3.43 . \mathrm{a})$ is nothing but the simplified equation of

$$
y^{(4)}=5 \frac{y^{\prime} y^{\prime \prime \prime}}{y}-5\left[\frac{\left(y^{\prime}\right)^{2}}{y^{2}}-\nu^{2} y^{2}\right] y^{\prime \prime}-5 \nu^{2} y\left(y^{\prime}\right)^{2}-\nu^{4} y^{5}+z y+1
$$

Equation (3.44) was given in [17], and there exists Bäcklund transformation between (3.44) and

$$
v^{(4)}=-5 v^{\prime} v^{\prime \prime}+5 v^{2} v^{\prime \prime}+5 v\left(v^{\prime}\right)^{2}-v^{5}+z v+(\nu+1),
$$

which was first given in the work of A.N.W. Hone [12], studied in [23, 24] and also proposed as defining a new transcendent $[6,9]$. Moreover (3.45) can be obtained as the similarity reduction of the modified Sawada-Kotera (mSK) equation [12].

When $k=3$, (3.43.a) gives the simplified equation of

$$
\begin{aligned}
y^{(4)} & =5 \frac{y^{\prime} y^{\prime \prime \prime}}{y}+\frac{5}{2} \frac{\left(y^{\prime \prime}\right)^{2}}{y}-\left[\frac{25}{2} \frac{\left(y^{\prime}\right)^{2}}{y^{2}}-\frac{5}{2} \nu^{2} y^{2}+\beta\right] y^{\prime \prime}+\frac{45}{8} \frac{\left(y^{\prime}\right)^{4}}{y^{3}}-\left[\frac{5}{4} \nu^{2} y^{2}-\frac{3}{2} \beta\right] \frac{\left(y^{\prime}\right)^{2}}{y} \\
& -\frac{3}{8} \nu^{4} y^{5}+\frac{1}{2} \beta \nu^{2} y^{3}+z y+2 \epsilon, \quad \epsilon= \pm 1 .
\end{aligned}
$$


Equation (3.46) was introduced in [17], and there exists Bäcklund transformation between (3.46) and the second member of the generalized second Painlevé equation, $\mathrm{P}_{\mathrm{II}}[6,9,21]$

$$
v^{(4)}=10 v^{2} v^{\prime \prime}+10 v\left(v^{\prime}\right)^{2}-6 v^{5}-\beta\left(v^{\prime \prime}-2 v^{3}\right)+z v+\nu .
$$

(3.47) can be obtained as the similarity reduction of the fifth order mKdV equation [12]. Moreover, when $k=3$, if one lets $y=1 / u$ then (3.43.a) gives the simplified equation of

$$
\begin{aligned}
u^{(4)}= & 3 \frac{u^{\prime} u^{\prime \prime \prime}}{u}+\frac{7}{2} \frac{\left(u^{\prime \prime}\right)^{2}}{u}-\frac{17}{2} \frac{\left(u^{\prime}\right)^{2} u^{\prime \prime}}{u^{2}}+\frac{27}{8} \frac{\left(u^{\prime}\right)^{4}}{u^{3}}-\left(\beta-\frac{5 \delta}{u^{2}}\right) u^{\prime \prime} \\
& +\frac{1}{2}\left(\frac{\beta}{u}-\frac{15 \delta}{u^{3}}\right)\left(u^{\prime}\right)^{2}-2 \nu u^{2}+2 \alpha z u-\frac{\beta \delta}{u}+\frac{3 \delta^{2}}{2 u^{3}} .
\end{aligned}
$$

Equation (3.48) was considered in [20]. When $k=7$, (3.43.a) gives the simplified equation of

$$
\begin{aligned}
y^{(4)} & =5 \frac{y^{\prime} y^{\prime \prime \prime}}{y}+\frac{15}{4} \frac{\left(y^{\prime \prime}\right)^{2}}{y}-\left[\frac{65}{4} \frac{\left(y^{\prime}\right)^{2}}{y^{2}}-\frac{5}{4} \nu^{2} y^{2}\right] y^{\prime \prime}+\frac{135}{16} \frac{\left(y^{\prime}\right)^{4}}{y^{3}}+\frac{5}{8} \nu^{2} y\left(y^{\prime}\right)^{2} \\
& -\frac{1}{16} \nu^{4} y^{5}+z y-2 .
\end{aligned}
$$

Equation (3.49) was introduced in [17], and there exists Bäcklund transformation between (3.49) and (3.45) with $\nu=\hat{\nu}-(3 / 2)$.

For all the other cases, the simplified equations are the same as the reduced equations (1.3) with the coefficients for $n=-1$.

\section{Leading order $\alpha=-4,-3$}

$\alpha=-4,-3$ are also a possible leading order of the equation (1.2) if $F=0$. For $\alpha=-4$, by adding terms of order -8 , we obtain the following simplified equation:

$$
y^{(4)}=a_{1} \frac{y^{\prime} y^{\prime \prime \prime}}{y}+a_{2} \frac{\left(y^{\prime \prime}\right)^{2}}{y}+a_{3} \frac{\left(y^{\prime}\right)^{2} y^{\prime \prime}}{y^{2}}+a_{4} \frac{\left(y^{\prime}\right)^{4}}{y^{3}}+d y^{5}
$$

where $d$ is a constant.

Suppose that (1.9), (1.10), (1.11), (1.18), (1.19), (1.20), (1.21), (1.22), (1.34), (1.35) and (1.36) hold, and substitute $y \cong y_{0}\left(z-z_{0}\right)^{-4}+\beta\left(z-z_{0}\right)^{r-4}$, into (4.1). Then we obtain the following equations for the Fuchs indices $r$ and $y_{0}$

$$
\begin{gathered}
Q(r)=(r+1)\left[r^{3}+\left(4 a_{1}-23\right) r^{2}-2\left(32 a_{1}+20 a_{2}+8 a_{3}-101\right) r\right. \\
+4\left[120 a_{1}+100 a_{2}+80 a_{3}+64 a_{4}-210\right]=0, \\
d y_{0}+4\left(120 a_{1}+100 a_{2}+80 a_{3}+64 a_{4}-210\right)=0,
\end{gathered}
$$

respectively [22]. (4.3) implies that, there is only one branch. Now, we determine $y_{0}$, and $d$ for each cases of $\left(a_{1}, a_{2}, a_{3}, a_{4}\right)$ such that the branch is the principal branch. If $r_{0}=-1$ and $r_{i}, i=1,2,3$ then (4.2) implies that

$$
\begin{gathered}
\sum_{i=1}^{3} r_{i}=-\left(4 a_{1}-23\right), \quad \sum_{\substack{i, j=1 \\
i \neq j}}^{3} r_{i} r_{j}=-2\left(32 a_{1}+20 a_{2}+8 a_{3}-101\right), \\
\prod_{i=1}^{3} r_{i}=-4\left(120 a_{1}+100 a_{2}+80 a_{3}+64 a_{4}-210\right)=d y_{0},
\end{gathered}
$$

provided that the right hand sides of (4.4) are positive integers. 
We have the following equations which have the additional leading terms: For the case 7, (1.21);

$$
\begin{aligned}
& y^{(4)}=\frac{7}{2} \frac{y^{\prime} y^{\prime \prime \prime}}{y}+2 \frac{\left(y^{\prime \prime}\right)^{2}}{y}-8 \frac{\left(y^{\prime}\right)^{2} y^{\prime \prime}}{y^{2}}+\frac{7}{2} \frac{\left(y^{\prime}\right)^{4}}{y^{3}}+24 y^{2}, \\
& y_{0}=1, \quad\left(r_{1}, r_{2}, r_{3}\right)=(2,3,4) .
\end{aligned}
$$

For the case 10, (1.35);

$$
\begin{aligned}
& y^{(4)}=3 \frac{y^{\prime} y^{\prime \prime \prime}}{y}+\frac{9}{4} \frac{\left(y^{\prime \prime}\right)^{2}}{y}-\frac{29}{4} \frac{\left(y^{\prime}\right)^{2} y^{\prime \prime}}{y^{2}}+\frac{49}{16} \frac{\left(y^{\prime}\right)^{4}}{y^{3}}+36 y^{2} \\
& y_{0}=1, \quad\left(r_{1}, r_{2}, r_{3}\right)=(2,3,6) .
\end{aligned}
$$

For all the other cases, $d=0$ and the simplified equations are the same as the reduced equations (1.3) with the coefficients $\left(a_{1}, a_{2}, a_{3}, a_{4}\right)$, for $n=-4$.

For $\alpha=-3$, the simplified equation is

$$
y^{(4)}=a_{1} \frac{y^{\prime} y^{\prime \prime \prime}}{y}+a_{2} \frac{\left(y^{\prime \prime}\right)^{2}}{y}+a_{3} \frac{\left(y^{\prime}\right)^{2} y^{\prime \prime}}{y^{2}}+a_{4} \frac{\left(y^{\prime}\right)^{4}}{y^{3}}+f y y^{\prime}
$$

where $f$ is a constant. Substituting $y \cong y_{0}\left(z-z_{0}\right)^{-3}+\beta\left(z-z_{0}\right)^{r-3}$, into (4.7) gives the following equations for the Fuchs indices $r$ and $y_{0}$

$$
\begin{gathered}
Q(r)=(r+1)\left[r^{3}+\left(3 a_{1}-19\right) r^{2}-3\left(13 a_{1}+8 a_{2}+3 a_{3}-46\right) r\right. \\
+9\left[20 a_{1}+16 a_{2}+12 a_{3}+9 a_{4}-40\right]=0 \\
f y_{0}-3\left(20 a_{1}+16 a_{2}+12 a_{3}+9 a_{4}-40\right)=0
\end{gathered}
$$

respectively. (4.9) implies that, there is only one branch. By following the same procedure, only for the case $10,(1.35)$ we obtain the following equation which has the principal branch and admits the additional leading term (i.e. $f \neq 0$ ).

$$
\begin{aligned}
& y^{(4)}=\frac{10}{3} \frac{y^{\prime} y^{\prime \prime \prime}}{y}+\frac{8}{3} \frac{\left(y^{\prime \prime}\right)^{2}}{y}-\frac{82}{9} \frac{\left(y^{\prime}\right)^{2} y^{\prime \prime}}{y^{2}}+\frac{112}{27} \frac{\left(y^{\prime}\right)^{4}}{y^{3}}+8 y y^{\prime}, \\
& y_{0}=-1, \quad\left(r_{1}, r_{2}, r_{3}\right)=(2,3,4) .
\end{aligned}
$$

For all the other cases, $f=0$ and the simplified equations are the same as the reduced equations (1.3) with the coefficients $\left(a_{1}, a_{2}, a_{3}, a_{4}\right)$, for $n=-3$.

\section{$5 \quad$ Negative resonances}

In the previous sections, we considered the case of existence of at least one principal branch and obtained the simplified equations. In this section, we present some of the simplified equations which admit the negative resonances. For the leading order $\alpha=-4$, and for the case 7 (1.21), case 8 (1.22), case 9 (1.34) and case 10 (1.35), we obtain the following simplified equations:

$$
\begin{aligned}
& y^{(4)}=5 \frac{y^{\prime} y^{\prime \prime \prime}}{y}+5 \frac{\left(y^{\prime \prime}\right)^{2}}{y}-17 \frac{\left(y^{\prime}\right)^{2} y^{\prime \prime}}{y^{2}}+8\left[\frac{\left(y^{\prime}\right)^{4}}{y^{3}}-y^{2}\right], \\
& y_{0}=21, \quad\left(r_{1}, r_{2}, r_{3}\right)=(-7,4,6), \\
& y^{(4)}=4 \frac{y^{\prime} y^{\prime \prime \prime}}{y}+3 \frac{\left(y^{\prime \prime}\right)^{2}}{y}-\frac{21}{2} \frac{\left(y^{\prime}\right)^{2} y^{\prime \prime}}{y^{2}}+\frac{9}{2} \frac{\left(y^{\prime}\right)^{4}}{y^{3}}-4 y^{2}, \\
& y_{0}=18, \quad\left(r_{1}, r_{2}, r_{3}\right)=(-3,4,6),
\end{aligned}
$$




$$
\begin{aligned}
& y^{(4)}=8 \frac{y^{\prime} y^{\prime \prime \prime}}{y}+6 \frac{\left(y^{\prime \prime}\right)^{2}}{y}-36 \frac{\left(y^{\prime}\right)^{2} y^{\prime \prime}}{y^{2}}+24\left[\frac{\left(y^{\prime}\right)^{4}}{y^{3}}-y^{2}\right], \\
& y_{0}=1, \quad\left(r_{1}, r_{2}, r_{3}\right)=(-2,-3,-4),
\end{aligned}
$$

and

$$
\begin{aligned}
& y^{(4)}=3 \frac{y^{\prime} y^{\prime \prime \prime}}{y}+\frac{7}{2} \frac{\left(y^{\prime \prime}\right)^{2}}{y}-\frac{17}{2} \frac{\left(y^{\prime}\right)^{2} y^{\prime \prime}}{y^{2}}+\frac{27}{8} \frac{\left(y^{\prime}\right)^{4}}{y^{3}}-8 y^{2}, \\
& y_{0}=18, \quad\left(r_{1}, r_{2}, r_{3}\right)=(-3,6,8),
\end{aligned}
$$

respectively. The canonical forms of the equations (5.2) and (5.4) were also given in [20]. If one lets $u=1 / y$, equation (5.3) gives $u^{(4)}=24$.

For the leading order $\alpha=-3$, and for the case 4 (1.18), and case 10 (1.35), we obtain the following simplified equations:

$$
\begin{aligned}
& y^{(4)}=7 \frac{y^{\prime} y^{\prime \prime \prime}}{y}+6 \frac{\left(y^{\prime \prime}\right)^{2}}{y}-30 \frac{\left(y^{\prime}\right)^{2} y^{\prime \prime}}{y^{2}}+18 \frac{\left(y^{\prime}\right)^{4}}{y^{3}}-6 y y^{\prime}, \\
& y_{0}=1, \quad\left(r_{1}, r_{2}, r_{3}\right)=(-3,-2,3),
\end{aligned}
$$

and

$$
\begin{aligned}
& y^{(4)}=2 \frac{y^{\prime} y^{\prime \prime \prime}}{y}+4 \frac{\left(y^{\prime \prime}\right)^{2}}{y}-2 \frac{\left(y^{\prime}\right)^{2} y^{\prime \prime}}{y^{2}}+6 y y^{\prime} \\
& y_{0}=20, \quad\left(r_{1}, r_{2}, r_{3}\right)=(-5,6,12),
\end{aligned}
$$

respectively.

For the leading order $\alpha=-2$, single branch, i.e. $b_{3}=0$, the case $2(1.10)$, and case $7(1.21)$ lead to the following simplified equations:

$$
\begin{aligned}
& y^{(4)}=6 \frac{y^{\prime} y^{\prime \prime \prime}}{y}+5 \frac{\left(y^{\prime \prime}\right)^{2}}{y}-22 \frac{\left(y^{\prime}\right)^{2} y^{\prime \prime}}{y^{2}}+12 \frac{\left(y^{\prime}\right)^{4}}{y^{3}}-2 y y^{\prime \prime}, \\
& y_{0}=1, \quad\left(r_{1}, r_{2}, r_{3}\right)=(-2,2,3),
\end{aligned}
$$

and

$$
\begin{aligned}
& y^{(4)}=5 \frac{y^{\prime} y^{\prime \prime \prime}}{y}+5 \frac{\left(y^{\prime \prime}\right)^{2}}{y}-17 \frac{\left(y^{\prime}\right)^{2} y^{\prime \prime}}{y^{2}}+8 \frac{\left(y^{\prime}\right)^{4}}{y^{3}}-2\left[y y^{\prime \prime}+\left(y^{\prime}\right)^{2}\right] \\
& y_{0}=1, \quad\left(r_{1}, r_{2}, r_{3}\right)=(-2,2,5)
\end{aligned}
$$

respectively.

In conclusion, we introduced the simplified equations of non-polynomial fourth order equations with the leading orders $\alpha=-4,-3-2,-1$, such that all of which pass the Painlevé test. More over the compatibility conditions corresponding the parametric zeros; that is, the compatibility conditions at the resonances of the equations obtained by the transformation $y=1 / u$ are identically satisfied. The corresponding simplified equation to (1.2) can be obtained by differentiating twice the leading terms of the third (or fourth) Painlevé equation and adding the terms of order -5 or greater as $z \rightarrow z_{0}$ with constant coefficients such that, $y=0, \infty$ are the only singular values of equation in $y$, and they are of order $\epsilon^{-4}$ or greater, if one lets $z=\zeta_{0}+\epsilon t$. Hence, these equations can be considered as the generalization of the third (or fourth) Painlevé equation.

In the second, third and fourth sections, we investigated the cases of leading order $\alpha=$ $-2,-1$ and $\alpha=-4,-3$ respectively with the condition of the existence of at least one principal branch. But, in the case of more than one branch, the compatibility conditions at the positive resonances for the second, third and fourth branches are identically satisfied for each cases. 
For the case of $\alpha=-4,-3,-2$ the simplified equations are examined without any restriction. For the case of $\alpha=-1, c_{1}=0$, and single and double branch cases were examined without any additional condition. All the other subcases of the case $\alpha=-1$ were investigated for some particular values of $n$. Some of the equations presented in these sections were considered in the literature before. In the last section, instead of having positive distinct integer resonances, we considered the case of distinct integer resonances. In this case, for the leading order $\alpha=-4,-3$ and $\alpha=-2$ single branch case was considered. Canonical forms of some of the equations given in the last section were introduced in the literature before. The canonical form of all the given simplified equations can be obtained by adding appropriate non-dominant terms with the coefficients analytic in $z$. The coefficients of the non-dominant terms can be determined from the compatibility conditions at the resonances and from the compatibility conditions corresponding the parametric zeros.

\section{References}

[1] P. Painlevé, Memoire sur les équations différentielles dont l'intégrale générale est uniforme, Bull. Soc. Math. France, 28 (1900), 201-261

P. Painlevé, Sur les équations différentielles du second ordre et d'ordre supérieur dont l'intégrale générale est uniforme, Acta. Math. 25 (1902), 1-85 .

[2] B. Gambier, Sur les équations différentielles du second ordre et du premeir degré dont l'intégrale générale est a points critiques fixés, Acta. Math. 33 (1909), 1-55 .

[3] E.L. Ince, Ordinary Differential Equations, Dover, New York 1956.

[4] F. Bureau, Differential equations with fixed critical points, Ann. Math. Pura Appl. (IV), 66 (1964), 1-116.

[5] U. Muğan and F. Jrad, Painlevé test and the first Painlevé hierarchy, J. Phys. A:Math. Gen. 32 (1999), 7933-7952.

[6] U. Muğan and F. Jrad, Painlevé test and higher order differential equations, J. Nonlinear Math. Phys. 9, Nr.3 (2002), 282-310.

[7] N.A. Kudryashov, The first and second Painlevé equations of higher order and some relations between them, Phys. Lett. A 224 (1997), 353-360.

[8] A.P. Clarkson, N. Joshi and A. Pickering, Bäcklund transformations for the second Painlevé hierarchy: a modified truncation approach, Inverse Problems, 15 (1999), 175187.

[9] C. M. Cosgrove, Higher-order Painleve equations in the polynomial class I. Bureau symbol P2, Stud. Appl. Math. 104, Nr.1 (2000), 1-65.

C. M. Cosgrove, Higher order Painlevé equations in the polynomial class II, Bureau symbol P1, Preprint, University of Sydney, School of Mathematics and Statistics, Nonlinear Analysis Research Reports 2000-06.

[10] N.A. Kudryashov, Some fourth-order ordinary differential equations which pass the Painlevé test, J. Nonlin. Math. Phys., 8 (2001) 172-177

[11] C. M. Cosgrove, Chazy Classes IX-XI of Third Order Diffrential Equations, Stud. Appl. Math. 104, Nr. 3 (2000), 171-228. 
[12] A.N.W. Hone, Non-autonomous Hénon-Heiles sytems, Physica D 118, (1998), 1-14.

[13] J. Chazy, Sur les équations différentielles du troisieme et d'ordre supérieur dont l'intégrale générale a ses points critiques fixés, Acta Math. 34 (1911), 317-385.

[14] H. Exton, Non-linear ordinary differential equations with fixed critical points, Rend. Mat. 6, Nr.2: (1973), 419-462.

[15] I. P. Martynov, Third order equations with no moving critical singularities, Differents. Uravn. 21, Nr.6 (1985), 937-946.

[16] U. Muğan and F. Jrad, Non-polynomial third order equations which pass the Painlevé test, Z.Naturforsch. A, 59a, (2004) 163-180.

[17] P.R. Gordoa, U.Muğan, A.Pickering and A.Sakka, Bäcklund transformations for higher Painlevé equations, Chaos, Solitons and Fractals, 22 (2004) 1103-1115.

[18] N.A. Kudryashov, Painlevé property and the first integrals of nonlinear differential equations, arXiv:nonlin.SI/0408041,

N.A. Kudryashov, Fuchs indices and the first integrals of nonlinear differential equations, Chaos, Solitons and Fractals, in press.

[19] A.V. Kitaev, Caustics in 1+1 integrable systems, J. Math. Phys., 35 (1994) 2934-2954.

[20] N.A. Kudryashov, Fourth-order analogies to the Painlevé equations, J. Phys. A: Math. Gen., 35, (2002), 4617-4632.

[21] A. Pickering, Coalescence limits for higher order Painlevé equations, Phys. Lett A, (2002) $275-280$.

[22] M.J. Ablowitz, A. Ramani and H. Segur, Nonlinear evolution equations and ordinary differential equations of Painlevé type, Lett. Nuovo Cim. 23 (1978), 333-338.

[23] N.A. Kudryashov, Transcendents defined by nonlinear fourth-ordr ordinary differential equations, J. Phys. A: Math. Gen., 32, (1999), 999-1013.

[24] N.A. Kudryashov, Two hierarchies of ordinary differential equations and their properties, Phys. Lett. A, 252, (1999), 173-179. 